\title{
Research on Fault of Distribution Network based on Structure Matrix
}

\author{
Ying Cao \\ Northeast Petroleum University \\ Qinhuangdao,Hebei, 066004 China
}

\begin{abstract}
This paper analyzes the existing matrix algorithm and the advantages and disadvantages of fault location in distribution network, integrated several fault location algorithm based on matrix structure, and proposed an improved matrix algorithm. Improved algorithm overcomes the judgment of peripheral fault power failure, line defects structure matrix cannot be based on complex networks, multiple faults more accurately judge the fault of power supply, and the principle is dear, there is no false positive and false negative phenomenon, a small amount of computation, fast reaction speed, strong adaptability to practical engineering. It overcomes the structure matrix that cannot judge the power failure and line endings faults, So it can accurate judgment the fault of supply multiple faults and more complex network, and clear principle, there is no false positive and false negative phenomenon, and have a small amount of computation, fast reaction speed, strong adaptability to practical engineering.
\end{abstract}

Keywords: Power distribution network; fault location; matrix algorithm;

\section{I.INTRODUCTION}

Electricity distribution network is a link that power system develop, change, send, and breeding last of supply to consumers. With the continuous improvement of people's material and cultural living standards, users of power quality and reliability requirements are getting higher and higher, even momentary power interruption cannot stand. In recent years, some scholar use genetic algorithms, Petri net theory, and thermal arc search distribution network fault location. Which, because of its simple and intuitive matrix algorithm, small calculation, etc., may be widely applied.

In the distribution network, when a section occurs a fault, firstly accurately position the fault section, promptly switch off the section in order to isolate the fault and non-fault section; to restore power supply quickly, thus avoiding failure caused the overall loss of power line. When a fault occurs in distribution network. Real time information of each as sociated feeder monitoring terminal of the corresponding switches and contact switch to master station system by data communication, master station system according to the fault location algorithm of automatic positioning faulty segment, and issued the command to the associated feeder monitoring terminal operating switch device fault section isolation, and restore the power supply of non-fault section. It can make full use of computer technology, communication technology, information technology, to avoid multiple coincidence Substation Switching, reducing the fault influence scope, the whole process of rapid completion of fault location, isolation, load transfer, the network reconfiguration to restore power supply.

\section{II.STRUCT URE OF THE MONIT OR TERMINAL FOR DISTRIBUTION NETWORK}

Distribution network automation system of feeder monitoring terminal composed of a feeder monitoring terminal, communication network and master station system. Distribution network automation system is as shown in figure 1 based on GPRS wireless communication network, the master station of the system divided into 3 layers; data acquisition layer, data management layer and application layer. Data collection layer based on GPRS communication access feeder monitoring terminal, according to the IEC 60870-5-104 protocol analytical data and preliminary treatment, monitoring the quality of communication, manage ment communication resources. It mainly consists of communication access equipment, the communication server, support software, communication protocol analysis software. The data management layer are processed, classified storage of data collection, the establishment and management of distribution network fault location and integrated data platform, data exchange and interfaces with other systems. It mainly by the database server, the data storage and backup equipment, interface equipment as well as the database management software. According to the demand of application, we develop application software in the application layer development with support function data, such as a terminal management, alarm information management, graphics modeling, visualization of topology analysis, feeder fault section location, report management, system management etc..

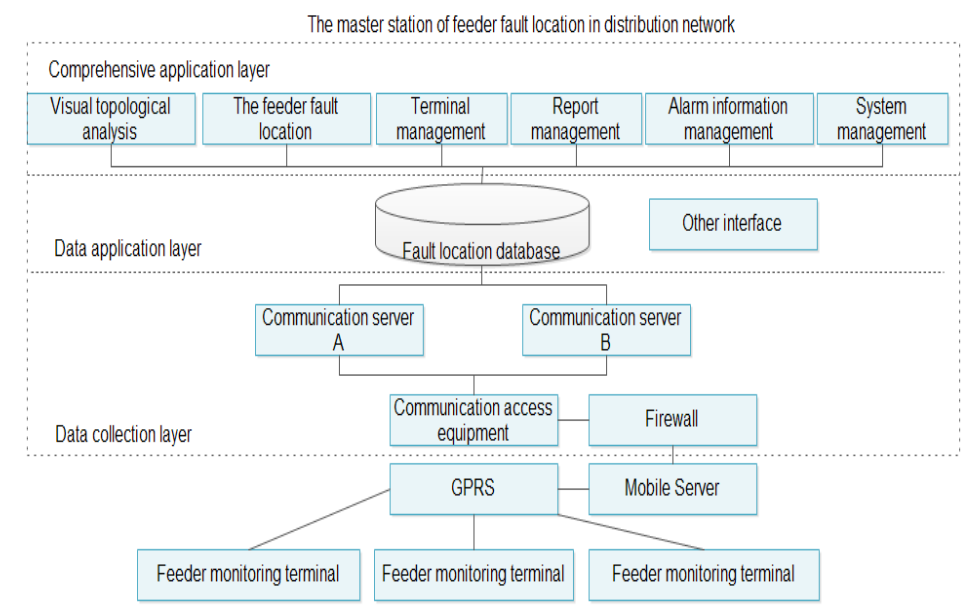

Fig.1 The systemstructure of distribution network 


\section{III.THE FAULT CLASSIFICATION OF DIST RIBUTION NETWORK}

Generally it can be divided into two types of fault in distribution network: the transient faults and permanent faults. Fault handling overhead lines need to distinguish the transient faults and permanent faults. For transient fault, be eliminated by a reclosing substation circuit breaker; and for permanent fault, reclosing failure, must carry on the distribution network. The smaller the possibility of transient faults occur cables, permanent fault is often need to be processed once failure.

For the radiation network, tree network and in the open loop operation of the ring network, fault section location only need to judge whether each switch along through fault current. If the line is single fault, load direction along the power to last between experienced fault current and the first experienced no fault current switch for fault section. In order to determine whether the fault current flowing through the switch, the need for the setting of each feeder monitoring terminal mounted thereon, not because of the difference between each switch setting to locate the fault section, so this setting more convenient.

In the closed loop operation of the ring network, it must be based on the flow in each switch fault power direction to fault section judgment. You must collect current and voltage signals at the same time. In order to determine whether the switch through the fault power, must also be set for each feeder monitoring terminal mounted thereon. In this case, when the segment switch through greater than the fault current setting value, a fault is indicated. Criterion for fault section: between the adjacent switch two fault power direction different sections is the fault section.

\section{IV.DIST RIBUTION NETWORK FAULT LOCATION ALGORITHM BASED ON MATRIX OPERATION}

1) The net shaped structure matrix $C$

Distribution network fault decision sector normally take open contact switch as the boundary point of the network, select only the interval containing the fault information calculation. The switches in the network, the circuit breaker and the contact switch as the key node numbering, and set the power positive direction: in the single power supply network, the natural direction line power is the positive direction of the feeder network; in power supply network, as suming the distribution network is composed of a power supply (the power supply choose any positive direction), feeder is the power to the power supply network. $\mathrm{N}$ nodes of the network, network structure matrix $\mathrm{C}$ is the $\mathrm{N} \times \mathrm{N}$ matrix, defines the elements are as follows: if i node has child nodes $\mathrm{J}$, cij $=1$, or $\mathrm{cij}=0$. That is, if the node $\mathrm{i}$ and node $\mathrm{j}$ has a positive direction feeder line and the feeder line is from node $i$ to node $j$, elements of $c i j=1$ network structure in the corresponding matrix $\mathrm{C}$, $\mathrm{cij}=0$. The network matrix $\mathrm{C}$ is non symmetric matrix, reflecting the real network topology.

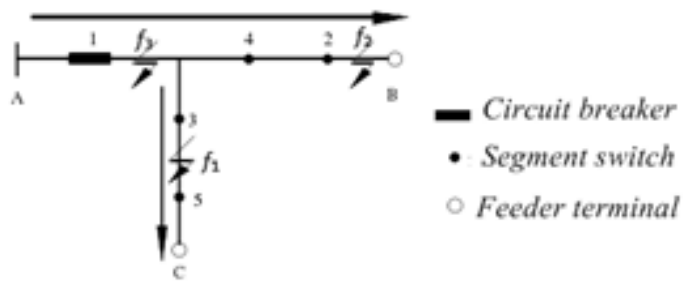

Fig.2 Simple feeder network

As shown in Figure 2, the net shaped structure matrix $\mathrm{C}$ feeder is:

$$
c=\left(\begin{array}{lllll}
0 & 0 & 1 & 1 & 0 \\
0 & 0 & 0 & 0 & 0 \\
0 & 0 & 0 & 0 & 1 \\
0 & 1 & 0 & 0 & 0 \\
0 & 0 & 0 & 0 & 0
\end{array}\right)
$$

2) Fault information matrix $G$

Fault information matrix is a network of $\mathrm{N}$ nodes, the corresponding $\mathrm{N} \times \mathrm{N}$ diagonal matrix. Set three working modes of $0,1,-1$ in the feeder terminal unit FTU [1]. The formation process of the corresponding elements are as follows: 0 for no fault current through, no need to send the fault signal, gij=0; (2) 1 representative the fault current through the current direction, and with the assumed power in the direction of $\mathrm{gjj}=1$; the -1 , representing the fault current through the current square to the contrary, and assumed that the power is direction, gij=-1. Fault information matrix is from the FTU transmitted to the SCADA system. Fault information matrix $G$ was shown in figure 1:

$G=\left(\begin{array}{lllll}1 & 0 & 0 & 0 & 0 \\ 0 & 0 & 0 & 0 & 0 \\ 0 & 0 & 0 & 0 & 0 \\ 0 & 0 & 0 & 0 & 0 \\ 0 & 0 & 0 & 0 & 0\end{array}\right)$

3) Fault judgment matrix $P$

SCADA received the net shaped structure matrix $\mathrm{C}$ and fault information matrix $\mathrm{G}$, and add two together, to form the fault interval judgment matrix $\mathrm{P}$, namely $\mathrm{P}=\mathrm{C}+\mathrm{G}$. In Figure 1, by calculation, fault interval judgment matrix $\mathrm{P}$ :

$$
p=\left(\begin{array}{lllll}
1 & 0 & 1 & 1 & 0 \\
0 & 0 & 0 & 0 & 0 \\
0 & 0 & 0 & 0 & 1 \\
0 & 1 & 0 & 0 & 0 \\
0 & 0 & 0 & 0 & 0
\end{array}\right)
$$

4) Criterion of fault section location

According to the fault interval judgment matrix $\mathrm{P}$ can identify the fault section, specific criteria are as follows:

(1) when the fault occurs between the node $\mathrm{i}$ and node $\mathrm{j}$, the discriminant condition follow two: when pii $=1$, for all pij=1 in $\mathrm{J}(\mathrm{J}=\mathrm{I})$, all $\mathrm{pjj}=-1$ or $\mathrm{pjj}=0$; when $\mathrm{pii}=-1$, for all $\mathrm{pji}=-1$ in $\mathbf{J}(\mathrm{J} \neq \mathrm{I})$, there are $\mathrm{pjj}=0$. In fault localization process, meet any one, is judge fault exists between nodes.

(2) When the fault occurred in the end, the fault matrix must satisfy: pii=1, for all PIJ ( $\mathbf{J}=\mathrm{I})$ is 0 . For example, fault judgement matrix: 


$$
p=\left(\begin{array}{lllll}
1 & 0 & 1 & 1 & 0 \\
0 & 0 & 0 & 0 & 0 \\
0 & 0 & 0 & 0 & 1 \\
0 & 1 & 0 & 0 & 0 \\
0 & 0 & 0 & 0 & 0
\end{array}\right)
$$

Criterion analysis: (1) p $11=1, \mathrm{p} 13=1, \mathrm{p} 14=1, \mathrm{p} 33=0$, $\mathrm{p} 44=0$, meet the criterion (1), can determine the node failure occurred in 1, 3, 4; (2) $\mathrm{p} 44=0, \mathrm{p} 42=1$, and $\mathrm{p} 44=0$, and the node 2 to the end node, meet the criterion (2), can determine the end node 2 after a fault occurs; (3) $\mathrm{p} 55=0$, p $35=1$, and p $33=0$, meet the criterion (1), can determine the node 3 and node 5 fault occurs.

Example analysis of complex calculation:
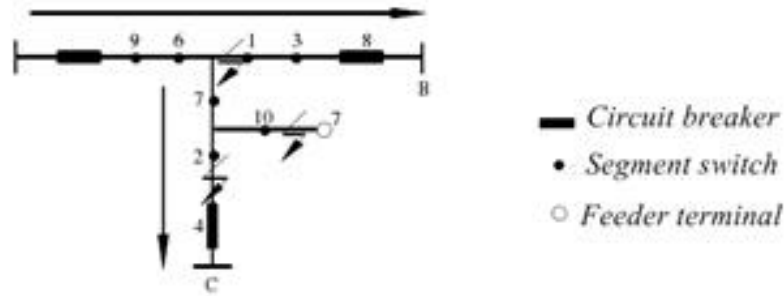

Fig.3 Complex feeder network

Study of power in Figure 3, complex feed network mode of mu ltiple faults under multi branch parallel power supply, without loss of generality, in order to upset. Figure A assuming power for the network only supply, positive direction of current is in the direction of the arrow diagram. In order to universality, practicability that algorithm, illustrations are respectively arranged 3 fault types: node 2 and node between the 4 line fault; node 1 , node 6 and node regional fault between 5 and 10; the end node fault.

Construct the net shaped structure matrix $\mathrm{C}$, and fault information matrix:

$C=\left(\begin{array}{cccccccccc}0 & 0 & 1 & 0 & 0 & 0 & 0 & 0 & 0 & 0 \\ 0 & 0 & 0 & 1 & 0 & 0 & 0 & 0 & 0 & 0 \\ 0 & 0 & 0 & 0 & 0 & 0 & 0 & 1 & 0 & 0 \\ 0 & 0 & 0 & 0 & 0 & 0 & 0 & 0 & 0 & 0 \\ 0 & 0 & 0 & 0 & 0 & 0 & 0 & 0 & 1 & 0 \\ 1 & 0 & 0 & 0 & 0 & 0 & 1 & 0 & 0 & 0 \\ 0 & 1 & 0 & 0 & 0 & 0 & 0 & 0 & 0 & 0 \\ 0 & 0 & 0 & 0 & 0 & 0 & 0 & 0 & 0 & 0 \\ 0 & 0 & 0 & 0 & 0 & 1 & 0 & 0 & 0 & 0 \\ 0 & 0 & 0 & 0 & 0 & 0 & 0 & 0 & 0 & 0\end{array}\right) G=\left(\begin{array}{cccccccccc}-1 & 0 & 0 & 0 & 0 & 0 & 0 & 0 & 0 & 0 \\ 0 & 1 & 0 & 0 & 0 & 0 & 0 & 0 & 0 & 0 \\ 0 & 0 & -1 & 0 & 0 & 0 & 0 & 0 & 0 & 0 \\ 0 & 0 & 0 & 0 & 0 & 0 & 0 & 0 & 0 & 0 \\ 0 & 0 & 0 & 0 & 1 & 0 & 0 & 0 & 0 & 0 \\ 0 & 0 & 0 & 0 & 0 & 1 & 0 & 0 & 0 & 0 \\ 0 & 0 & 0 & 0 & 0 & 0 & 1 & 0 & 0 & 0 \\ 0 & 0 & 0 & 0 & 0 & 0 & 0 & -1 & 0 & 0 \\ 0 & 0 & 0 & 0 & 0 & 0 & 0 & 0 & 1 & 0 \\ 0 & 0 & 0 & 0 & 0 & 0 & 0 & 0 & 0 & 1\end{array}\right)$

So. $\mathrm{P}=\mathrm{C}+\mathrm{G}$, get:

$$
P=C+G=\left(\begin{array}{cccccccccc}
-1 & 0 & 1 & 0 & 0 & 0 & 0 & 0 & 0 & 0 \\
0 & 1 & 0 & 1 & 0 & 0 & 0 & 0 & 0 & 0 \\
0 & 0 & -1 & 0 & 0 & 0 & 0 & 1 & 0 & 0 \\
0 & 0 & 0 & 0 & 0 & 0 & 0 & 0 & 0 & 0 \\
0 & 0 & 0 & 0 & 1 & 0 & 0 & 0 & 1 & 0 \\
1 & 0 & 0 & 0 & 0 & 1 & 1 & 0 & 0 & 0 \\
0 & 1 & 0 & 0 & 0 & 0 & 1 & 0 & 0 & 0 \\
0 & 0 & 0 & 0 & 0 & 0 & 0 & -1 & 0 & 0 \\
0 & 0 & 0 & 0 & 0 & 1 & 0 & 0 & 1 & 0 \\
0 & 0 & 0 & 0 & 0 & 0 & 0 & 0 & 0 & 1
\end{array}\right)
$$

Through the judgment of fault information in P: (1) $\mathrm{P} 22=1, \mathrm{P} 24=1, \mathrm{P} 44=0$ criterion is satisfied (1), can determine the node 2 and node failure occurs between the 4; (2) $\mathrm{P} 66=1, \mathrm{P} 61=1, \mathrm{P} 67=1, \mathrm{P} 11=-1, \mathrm{P} 77=1$, meet the criterion (1), can determine the fault in node 1 , node 6 and node 7. Region; (3) $P 10,10=1, P 10, j=0$ ( $j=0,1,3 \ldots$ 9), meet the criterion (2) at the end of the criterion, then judge the fault node 10 .

4 The process of fault location algorithm

When FTU detects the fault current, fault information will be transmitted to the FTU SCADA system. Calculating and judging the system failure to receive information of computer fault according to the preprogrammed, fault diagnosis is completed, a fault tripping signal corresponding to FTU, FTU to complete the trip operation. The programming process is shown in figure 4:

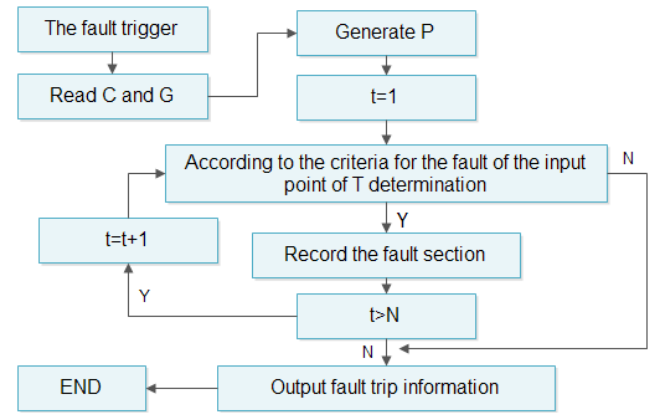

Fig.4 The flow chart of fault positioning algorithm

\section{CONCLUSIONS}

This paper analyzes the existing matrix algorithm based on advantages and disadvantages of fault location in distribution network, integrated net base matrix of several fault location algorithm, and proposed an improved algorithm of matrix. It overcomes the net base matrix cannot judge the peripheral fault line power failure, the disadvantages of complex networks, multiple faults more accurately judge the failure of power supply, and the principle is clear, there is no false positive and false negative phenomenon, and have a small amount of computation, fast reaction speed, strong adaptability to practical engineering.

\section{REFERENCE :}

[1] Ma Qiang, Zhang Limin, Liu Haoming. General matrix algorithm for fault section judgement[J]. power system protection and control, 2009, 37 (5) 14-17.

[2] Zhang Hong, Guo Zongren. Method of distribution network topology equivalent decoupling [J]. Grid technology 2004, 28 (15): 88-91.

[3] Su Yongzhi, Pan Zhencun, Ding Lei.A fast algorithm for complex distribution network fault location[J].Grid technology 2005, 29 (18): 75-78.

[4] Jun Fei, Shan Yuanda. Study of automatic fault location system in the distribution network[J],China Journal of electrical engineering 2000, 20 (9): 32-35.

[5] Huang. Simple matrix algorithm for fault location in distribution network[J]. Hubei electric power, 2005, 29 (5): 1-3. 